\title{
Resolution in patient data
}

\author{
Arnold G Vulto
}

The 17th European Association of Hospital Pharmacist (EAHP) Congress that was held in Milan in March this year was in many respects an exciting event. It was a huge social event with some 3700 attendants and the content reached a new high in number of sessions and quality. However, if you were among the many colleagues who stayed at home to look after our patients, you do not have to miss out because most sessions will be available soon in video format from the EAHP website (http://www.eahp.eu).

\section{We bring the congress to you}

In the European Journal of Hospital Pharmacy (EJHP) we try to cover the Congress as extensively as possible. In this issue, Professor Umberto Veronesi shares with us 50 years of experience in oncology care, and how this has shaped oncology pharmaceutical care today. I found it impressive to see such an icon of modern medicine placing the patient at the centre of treatment rather than either the doctor's priorities, as we often see, or the drug treatment, which is usually the pharmacist's focus. With his concluding list of roles for the hospital pharmacist, he connected flawlessly with the overall theme of the Congress: Special patient groups.

The idea of patients taking more responsibility for their own treatment is the subject of the report from Marcolongo and Ané on 'Therapeutic patient education'. Proper education of patients will improve self-management, adherence and overall quality of treatment, and has also been shown to be a powerful measure in reducing medication errors. It was interesting to see how these authors advocate a role for the pharmacist as a 'mediator', staying beside a patient, rather than a care provider, imposing on patients a treatment 'in their best interest'.

In the general congress report we elaborate on the two other plenary keynotes. Professor Faber from Copenhagen, Denmark presented an impressive lecture on risk management from a society perspective. The conference was concluded by Dr Thürman from Wuppertal, Germany,

Correspondence to Dr Arnold G Vulto, Department of Hospital Pharmacy, Erasmus University Medical Center, Gravendijkwal 230, 3015CE, Rotterdam, The Netherlands; editor.ejhp@bmjgroup.com providing an expert overview of current developments in geriatric pharmacotherapy, and how important hospital pharmacists are in the supervision of drug use in the growing population of older patients. She introduced compelling concepts, such as the pharmacological unhappy triad (diuretics plus non-steroidal anti-inflammatory drugs plus renin-angiotensin-aldosterone inhibitors) and potentially inappropriate medications (PIMs) for older people. Several lists of PIMs have been proposed and they can be included in our pharmacy expert systems when checking drug prescriptions, helping pharmacists to raise a red flag in time. Dr Thürman was critical of the evidence base for these lists because they have been based mostly on retrospective database analysis, linking drug use with some measure of adverse outcome, while prospective scientific evidence is still weak. Therein lies the attractiveness of a practicing clinician delivering a keynote; she could offer her own experience of several convincing patient cases, showing the usefulness of the concept. Watch the video once it comes online!

\section{Congress poster prize}

It is also worth mentioning the abstract that won the EAHP Poster Prize: Dr Jesse Swen from Leiden University Medical Center, The Netherlands, with a study building on earlier identification of alleles that increase the risk of type 2 diabetes mellitus. Dr Swen and his colleagues were able to calculate a diabetes risk score based on the number of increased risk alleles in a given patient. Subsequently they hypothesised that the higher the calculated risk based on genotyping 20 single-nucleotide polymorphisms, the more difficult it would be to stabilise blood sugars with sulfonylurea drugs. A nice example of how the genetic code - as also discussed in the first keynote from Professor Veronesi may affect success in drug treatment, and the EAHP Scientific Committee is signalling this as an important new development.

\section{Pharmacogenetic incidentalome}

We can expect a flood of similar findings as a result of the ease by which we can now decode a patient's DNA, and even determine which genes are more or less active in a certain disease. The treatment of HerNeu2positive breast cancers with trastuzumab has been mentioned previously in EJHP (see cover stories in EJHP 2011, issue 5) ${ }^{12}$, and now an international consortium led by Professor Carlos Caldas (Cambridge, UK) has been able to construct a prognostic 10-group classification of breast cancer by analysing the gene activity in 2000 patients with breast cancer (Nature, published online 18 April 2012). ${ }^{3}$ However, a month before the Cambridge study was published online, another group of UK researchers published in the New England Journal of Medicine (8

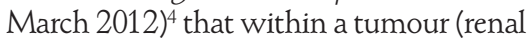
cancer in this case) considerable genetic variation may exist, actually hindering a personalised approach. This backs up Isaac S Kohane's comment in Nature Reviews Drug Discovery (February 2012): '(Mis) treating the pharmacogenetic incidentalome', in which he says it is too early to base therapy decisions on pharmacogenetic data because such data in many cases lack precision and confirmation.

How will we ever be able to achieve the resolution in patient data that is needed to provide personalised treatment? Every week new information is released on patient traits or biomarkers that we should consider when initiating, optimising and evaluating drug treatment. Most electronic prescribing systems have some kind of provision to check dosing and interactions and possibly also integrating renal function, but these systems will be slow to incorporate new findings and they have only limited potential to define special patients groups. We need powerful tools to integrate sparse data in our treatment decisions. Our memories are just not good enough and do not work that reliably.

Also in this issue, our Belgian colleagues have done an excellent job in providing us with a broad update on hospital pharmacy in Belgium. The recent overhaul of the hospital pharmacy training programme actually accommodates many of the demands that I have depicted above.

\section{Need for hospital pharmacy specialisation}

With the demands on hospital pharmacists to develop knowledge and skills, it is difficult to understand why there is only limited support for hospital pharmacy as a specialisation beyond the standard undergraduate curriculum as agreed under the Bologna umbrella. We need specialists that are able to understand new 


\section{Editorial}

developments in diagnosis and patient profiling, and translate them into practical treatment guidelines that can be understood by doctors, nurses and patients. The newly appointed EAHP Policy Officer, Richard Price, writes in the EAHP News section on the latest developments in the discussions EAHP has been having in Brussels to achieve mutual recognition for specialist hospital pharmacists. We need to find a poignant way to convince our politicians that without proper postgraduate training, pharmacists will not be able to provide an up-to-date standard of pharmaceutical care to their patients. And that in the end is the objective of conferences like the annual EAHP

Congress: better care for our patients.

Competing interests None.

Provenance and peer review Commissioned; internally peer reviewed.

European Journal of Hospital Pharmacy

2012;19:275-276.

doi:10.1136/ejhpharm-2012-000144

\section{References}

1. Metzger Filho O, Bozovic-Spasojevic I, Cardoso

F. Treatment of metastatic breast cancer: an overview. European Journal of Hospital Pharmacy: Practice 2011;17:20-23.
2. Metzger Filho O, Bozovic-Spasojevic I, Cardoso F. Treatment of early breast cancer: an overview. European Journal of Hospital Pharmacy: Practice 2011;17:11-18.

3. Curtis C, Shah SP, Chin S-F, et al. The genomic and transcriptomic architecture of 2,000 breast tumours reveals novel subgroups. Nature. Published Online First 18 April 2012. doi: 10.1038/nature10983.

4. Gerlinger M, Rowan AJ, Horswell S. Intratumor heterogeneity and branched evolution revealed by multiregion sequencing. New England Journal of Medicine 2012;366:883-92

5. Kohane IS. (Mis) treating the pharmacogenetic incidentalome. Nature Reviews Drug Discovery 2012;11:89-90. 